\title{
Numerical Simulation of Natural Air Convection in Inclined Eccentric Hemispheres Enclosure
}

\author{
Mariama Néné Koita1,2*, Mamadou Lamine Sow ${ }^{1,2}$, Oumar Dramé2, Babacar Mbow ${ }^{3}$, \\ Cheikh Mbow², Joseph Sarr ${ }^{1,2}$ \\ ${ }^{1}$ Groupe de Recherche sur l'Energie Solaire et les Transferts (GREST), Faculté des Sciences et Techniques, Université Cheikh \\ Anta DIOP (UCAD), Dakar, Sénégal \\ ${ }^{2}$ Laboratoire de Mécanique des Fluides, Faculté des Sciences et Techniques, Université Cheikh Anta DIOP (UCAD), Dakar, \\ Sénégal \\ ${ }^{3}$ Laboratoire de Physique des Semi-Conducteurs et D'énergie Solaire, Faculté des Sciences et Techniques, Université Cheikh \\ Anta DIOP (UCAD), Dakar, Sénégal \\ Email: *mariamanene.koita@ucad.edu.sn
}

How to cite this paper: Koita, M.N., Sow, M.L., Dramé, O., Mbow, B., Mbow, C. and Sarr, J. (2021) Numerical Simulation of Natural Air Convection in Inclined Eccentric Hemispheres Enclosure. Open Journal of Applied Sciences, 11, 722-735.

https://doi.org/10.4236/ojapps.2021.116053

Received: May 26, 2021

Accepted: June 26, 2021

Published: June 29, 2021

Copyright $\odot 2021$ by author(s) and Scientific Research Publishing Inc. This work is licensed under the Creative Commons Attribution International License (CC BY 4.0). http://creativecommons.org/licenses/by/4.0/ Open Access

\begin{abstract}
The subject of natural convection heat transfer is motivated by a wide range of applications in engineering technology. The hemispherical cavity is a part of basic geometries although it is not widely studied. The effect of inclinaison on natural convection fluid motions in the gap between two eccentric hemispheres is numerically studied. The inner hemisphere is subjected to a heat flux of a constant density and the outer one is maintened isothermal. The walls separating the two hemispheres are thermally adiabatic. Equations are formulated with vorticity and stream-functions variables. It is also assumed the fluid incompressible and obeys the approximation of Boussinesq. These equations are written by using bispherical coordinates system and solved by using a finite difference method. The results show the topology of flow is strongly dependent on the inclinaison because the flow can change from a unicellular regime to a multicellular regime by varying the inclination from 0 to $\pi$. By increasing the Rayleigh number $\left(10^{3}<R a<10^{7}\right)$, the flow intensifies. The results are shown in terms of streamlines and isotherms during their transient evolution.
\end{abstract}

\section{Keywords}

Bispherical Coordinates, Hemispherical Cavities, Rayleigh Number, Nusselt Number, Eccentricity, Streamlines, Isotherms

\section{Introduction}

Natural convection heat transfer in enclosures especially from objects limited by 
spheres surfaces is of considerable interest in many engineering areas. The spheres or hemispheres can be used as constructing and insulated surfaces The rectangular or square cavity has been extensively treated [1] [2] given their simplicity of implementation. Sarr et al. [3] and Waheed [4] studied the cylindrical annuli. Several analytic and numerical studies dealing with spherical sections have been presented by [5] [6]. However, very little works are done on natural convection between two hemispheres. Cabelli [7] examined the effect of inclinaison on the convective motion in hemispherical cavities. They showed that the axially symmetric motion which exists when the base of the hemisphere is horizontal becomes three-dimensional when the hemisphere is inclined. Shiina et al. [8] performed an experimental study of natural convection in a hemisphere that is differentially heated for nuclear applications. The heat transfer is empirically correlated by means of Nusselt-Rayleigh type. By studying theorical and experimental natural convection heat transfer from isothermal, Lewandowski et al. [9] presented their result in theorical consideration is presented in the Nusselt-Rayleigh correlation which the exponent of Rayleigh number is equal to a quarter. Saber et al. [10] numerically analysed the $2 \mathrm{D}$ convective in hemispherical cavities with planar inner surfaces. They observed that for differents boundaries temperatures conditions, the airflow in the cavities is monocellular and reaches steady state conditions for both cold and hot weather conditions. The numerical results are used to develop practical correlations for the Nusselt number. Bairi et al. [11] dealt with isothermal disks and dome faced downwards. Their results showed that the natural convection heat transfer is between $13 \%$ et $21 \%$ higher when the disk is maintened as compared to the case corresponding to imposed heat flux on the disk, for the same inclinaison angle and Rayleigh range. We note that all these hemispherical cavities mentioned have an enclosure formed by a dome and a horizontal plane. Little work has been done when the enclosure is delimited by two hemispheres. Thus, Alilat [12] studied the quantification of natural convection between two inclined concentric hemispheres for thermoregulation applications in electronics. His results showed that the inclinaison angle of the device affects only weakly the heat exchange and the natural convection heat transfers are also quantified by means of the Nusselt number. Recently, by studying natural convection between two eccentric hemispheres, Koita et al., [13] observed that the parameters of fluid depend strongly on the modified Rayleigh number as well as of the eccentricity. Also, the effects of eccentricity showed the center of rotation moves toward the top of enclosure when the eccentricity increases and the Nusselt number increases with the modified Rayleigh number. According to the authors' knowledge, transient natural convection between two eccentric inclined hemispheres has not been the subject of any study. The present numerical study is motivated by the interest to understand the effects of inclinaison, the eccentricity and the modified Rayleigh number on the convection motion over time between two hemispheres. The inner hemisphere is heated by a heat flux constant density and the outer one is maintened isothermal. The walls separating the two hemispheres are insulated. 


\section{Mathematical Formulation}

A schematic depiction of the problem is shown in Figure 1 contains a Newtonian fluid (air in this case) confined within enclosure delimited by two vertically eccentric hemispheres. Initially, the enclosure is at uniform temperature. From this time, the inner hemisphere of radius $R_{i}$ centered in $O_{i}$ is heated by a heat flux of constant density while the outer one of radius $R_{e}$ centered respectively on $O_{e}$ is maintened isothermal. The eccentricity $e^{\prime}$ is defined as the algebric distance separating the two centers of hemispheres. The walls separating the two domes ( $\theta=0$ and $\theta=\pi$ ) are insulated. The enclosure is inclined with respect to the vertical by an angle $\varphi$ from 0 to $\pi$. The two hemispheres being heated differently, a transient convection develops inside the enclosed space.

We considered the fluid Newtonian and the flow incompressible and bidimensional. Viscous dissipation, compressibility effects an thermal radiation are neglected. We further applied the Boussinesq approximation. An adapted conformal tranformation is used in order to reduce a curvilinear enclosure into a rectangular field. A bispherical coordinate system is chosen. The dimensionnal transformation from bispherical $(\eta, \theta)$ of Cartesian coordinate $(x, y)$ [14] is:

$$
\left\{\begin{array}{l}
x=a \frac{\sin \theta}{\cosh \eta-\cos \theta} \\
y=a \frac{\sinh \eta}{\cosh \eta-\cos \theta}
\end{array}\right.
$$

where $a$ is the parameter of torus pole.

The inner copula is materialized by line of coordinate $h=h_{i}$ and the outer hemisphere by $h=h_{e}$.

On the basis of these assumptions, the equations governing the problem in a dimensionless vorticity-stream function are written in bispherical coordinate. To transform these dimensional equations into a set of dimensionless equations, we used the references parameters as follow:

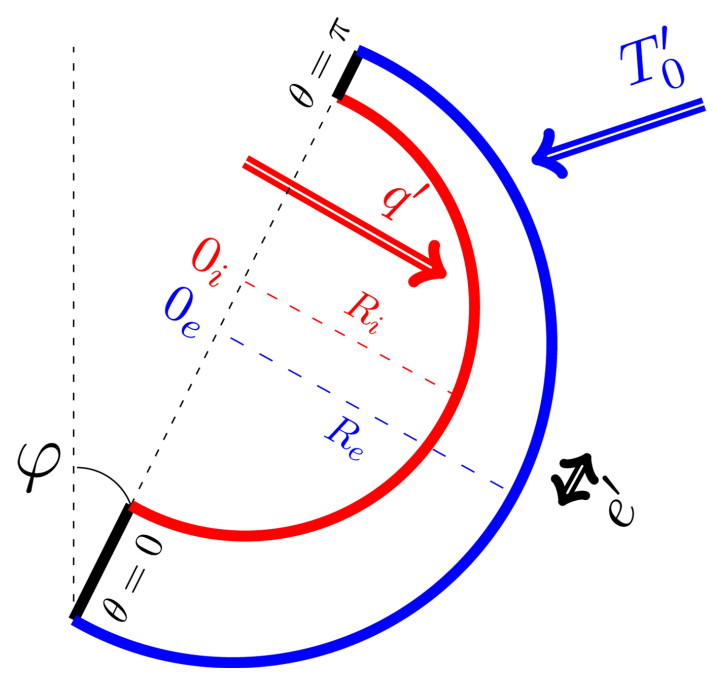

Figure 1. Schematic depiction of the physical model. 
$a, \frac{a^{2}}{\alpha}$ and $\frac{q a}{\lambda}$ which represent respectively the length, time and temperature gradient. Heat and momentum equations in the dimensionless form as given respectively as:

$$
\begin{gathered}
\partial_{t} T+\frac{1}{H}\left[U-\frac{g_{2}}{K}\right] \partial_{\eta} T+\frac{1}{H}\left[V+\frac{g_{1}}{K}\right] \partial_{\theta} T=\frac{1}{H^{2}}\left(\partial_{\eta}^{2} T+\partial_{\theta}^{2} T\right) \\
\partial_{t} \frac{\Omega}{K}+\frac{1}{H}\left[U-\frac{3 P r \cdot g_{2}}{K}\right] \partial_{\eta} \frac{\Omega}{K}+\frac{1}{H}\left[V+\frac{3 P r \cdot g_{1}}{K}\right] \partial_{\theta} \frac{\Omega}{K} \\
=\frac{P r}{H^{2}}\left[\partial_{\eta}^{2} \frac{\Omega}{K}+\partial_{\theta}^{2} \frac{\Omega}{K}\right]+\frac{R a \cdot P r}{K H}\left(G_{2 \varphi} \partial_{\eta} T-G_{1 \varphi} \partial_{\theta} T\right)
\end{gathered}
$$

where $\Psi$ and $\Omega$ are defined by:

$$
U=\frac{1}{K H} \partial_{\theta} \Psi, V=-\frac{1}{K H} \partial_{\eta} \Psi \text { and } \vec{\Omega}^{\prime}=\vec{\nabla} \wedge \vec{V}^{\prime} .
$$

With

$$
\begin{gathered}
\left\{\begin{array}{l}
g_{1}=g_{1}(\eta, \theta)=-\frac{1}{h} \partial_{\theta} k=\frac{1-\cos \theta \cosh \eta}{\cosh \eta-\cos \theta} \\
g_{2}=g_{2}(\eta, \theta)=\frac{1}{h} \partial_{\eta} k=-\frac{\sin \theta \sinh \eta}{\cosh \eta-\cos \theta}
\end{array}\right. \\
\left\{\begin{array}{l}
G_{1 \varphi}=G_{1}(\eta, \theta, \varphi)=g_{2} \cos \varphi+g_{1} \sin \varphi \\
G_{2 \varphi}=G_{2}(\eta, \theta, \varphi)=g_{1} \cos \varphi+g_{2} \sin \varphi
\end{array}\right.
\end{gathered}
$$

We defined the stream-function which intresically verifies incompressibility condition. His particularty is he has the dimension of a volume flow.

$$
\Psi=K \phi
$$

With $\phi$ is the stream-function which has a surface flow.

The dimensionless equation of stream-function is:

$$
\Omega=\frac{1}{K^{2} H}\left(G_{2 \varphi} \partial_{\eta} \Psi-G_{1 \varphi} \partial_{\theta} \Psi\right)-\frac{1}{K H^{2}}\left(\partial_{\eta}^{2} \Psi+\partial_{\theta}^{2} \Psi\right)
$$

These equations are subject to the following boundary conditions and initial:

- For $t=0$ :

$$
\Omega=\Psi=T=U_{\eta}=V_{\theta}=0
$$

- On the inner hemisphere $\left(\eta=\eta_{i}\right)$ :

$$
\begin{gathered}
U=V=\Psi=0 \\
\Omega=-\frac{1}{K H} \partial_{\eta}^{2} \Psi \\
\partial_{\eta} T=H_{i}
\end{gathered}
$$

- On the outer hemisphere $\left(\eta=\eta_{e}\right)$ :

$$
\begin{gathered}
U=V=\Psi=0 \\
\Omega=-\frac{1}{K H} \partial_{\eta}^{2} \Psi
\end{gathered}
$$

- On the vertical walls $(\theta=0)$ and $(\theta=\pi)$ 


$$
\begin{gathered}
U=V=\Psi=0 \\
\partial_{\theta} T=0
\end{gathered}
$$

The local and average Nusselt number are defined respectively by:

- For the inner hemisphere

$$
\begin{gathered}
N u_{i}=\frac{1}{T_{i, m}} \\
\overline{N u_{i}}=\frac{1}{S} \iint N u_{i} \mathrm{~d} S
\end{gathered}
$$

- For the inner hemisphere

$$
\begin{gathered}
N u_{e}=\frac{1}{H_{e} T_{i, m}} \partial_{\eta} T\left(\eta=\eta_{e}\right) \\
\overline{N u_{e}}=\frac{1}{S} \iint N u_{e} \mathrm{~d} S
\end{gathered}
$$

\section{Numerical Procedure}

Our physical domain is complex. the bispheric coordinate system we used allowed us to translate our curved walls into a parallelepiped grid. The implicit method alterning direction (A.D.I.) [15] is used for time integration with momentum and heat equations and the finite difference scheme is choosen for spatial integration. The system of linear equations obtained by using Thomas algorithm. The equations of stream-function is solved by using the Successive Over relaxation (S.O.R.) [16]. The convergence criterion as following is chosen for all variables dependant. It must be satisfied the following stop criterion:

$$
\sum_{i, j}\left(\left|\frac{w_{i, j}^{m+1}-w_{i, j}^{m}}{w_{i, j}^{m+1}}\right|\right) \leq 10^{-5}
$$

where $m$ denotes the iteration and $w$ stands for $\Psi, \Omega, T$.

\section{Computation Conditions}

Preliminary tests have been done on the influence on the mess and time step (see Table 1 and Table 2). We retain $51 \times 51$ for the grid system and $10^{-5}$ for the time which constitutes a good compromise between a precision and an acceptable computation time.

Table 1. Effects of time steps on the Nusselt number of the heat wall for $R a=10^{5}$, $e=0.5$ and $\varphi=\pi / 3$.

\begin{tabular}{cccc}
\hline & \multicolumn{3}{c}{ Time steps } \\
\cline { 2 - 4 } & $10^{-4}$ & $10^{-5}$ & $10^{-6}$ \\
\hline $\mathrm{Nu}$ & 3.2827 & 3.4581 & 3.8719 \\
$\begin{array}{c}\text { Time computing } \\
(\mathrm{min})\end{array}$ & 30 & 87 & 390 \\
$\bar{\delta}(\%)$ & 15.22 & 10.69 & 0 \\
\hline
\end{tabular}


Table 2. Effects of mesh refinement on the Nusselt number of the heat wall for $R a=10^{5}$, $e=0.6, \Delta t=10^{-5}$ and $\varphi=\pi / 3$.

\begin{tabular}{ccccccccc}
\hline & \multicolumn{8}{c}{ Meshgrid } \\
\cline { 2 - 8 } & $21 \times 21$ & $21 \times 41$ & $41 \times 41$ & $41 \times 51$ & $41 \times 81$ & $51 \times 51$ & $51 \times 81$ & $81 \times 81$ \\
\hline $\mathrm{Nu}$ & 3.1203 & 3.2509 & 3.2273 & 3.2220 & 3.2130 & 3.2173 & 3.2061 & 3.1985 \\
$\begin{array}{c}\text { Time } \\
\text { computing }\end{array}$ & 14 & 15 & 45 & 55 & 88 & 85 & 133 & 334 \\
$\bar{\delta}(\%)$ & 2.44 & 1.64 & 0.9 & 0.73 & 0.45 & 0.59 & 0.24 & 0 \\
\hline
\end{tabular}

Table 3 shows comparaison between the average Nusselt numbers of the inner wall for different Rayleigh numbers with others authors. Calculations are made with Prandlt number of air and with an inclinaison angle $\varphi=0$. The difference $(\bar{\delta})$ is less than $3.7 \%$ which shows a very good accord between their results.

$$
\text { With } \bar{\delta}=\frac{\left|N u_{\text {ref }}-N u_{\text {present study }}\right|}{N u_{\text {ref }}} \text {. }
$$

\section{Results and Discussion}

\subsection{Influence of Rayleigh Number}

Figures 2-5 present the evolution of the streamlines and isotherms over time respectively for $R a=10^{3}, R a=10^{5}, R a=10^{6}$ and $R a=10^{7}$. For $10^{3}$, at the first moments, the streamlines the flow is constitued by two cells that turns in opposite directions. The secondary cell disappears in favor of the main cell. The isotherms similar to eccentric circles marrying the internal wall at the first moments and are then slightly deformed at the beginning of time for $R a=10^{3}$ due to the phenomena of conduction as we can see it on Figure 2. Also For $R a=10^{5}$ and $R a=10^{6}$, we note a significant deformation of the isotherms reflecting the intensification of the convective effects. The secondary cell rather disappears compared to Figure 2. At $R a=10^{7}$, we are witnessing a strongly destabilized regime results in a multicellular flow. These results are in perfect agreement with those [6] [13] [17]. Indeed, when the Rayleigh number is less $10^{3}$, the flow is dominated by the conduction, when $R a=10^{5}$ or $10^{6}$, the convection predominates and when $R a$ reached the value $10^{7}$, we notice the formation of multi-cell flow.

\subsection{Inuence of the Angle}

Figures 6-9 depicted the evolution of streamlines and isotherms for various inclinaison angles $(\varphi=0, \pi / 3, \pi / 2,2 \pi / 3$ and $\pi)$. When the Rayleigh number is fixed at $10^{5}$ and the eccentricity at -0.5 . We notice that streamlines for configurations $\varphi=0$ and $\varphi=\pi$ (Figure 6 and Figure 10) has a unicellular flow what is in agreement with those of [13]. The isotherms are increasingly deformed over time. This deformation is more important with the configuration $\varphi=\pi$ where 
Table 3. Comparaison of the average Nusselt number of the hot wall with others authors.

\begin{tabular}{cccccc}
\hline & \multicolumn{5}{c}{$\mathrm{Ra}$} \\
\cline { 2 - 5 } & $10^{3}$ & $10^{4}$ & $10^{5}$ & $10^{6}$ & $10^{7}$ \\
\hline Present results & 2.125 & 3.0651 & 4.982 & 7.6874 & 11.671 \\
{$[6]$} & 2.062 & 3.062 & 4.977 & 7.7720 & 12.109 \\
$\bar{\delta}(\%)$ & 3.54 & 0.10 & 0.10 & 0.42 & 3.62 \\
{$[17]$} & 2.098 & 3.15 & 5.034 & 7.794 & 12.109 \\
$\bar{\delta}(\%)$ & 1.76 & 2.70 & 1.03 & 1.37 & 3.62 \\
\hline
\end{tabular}
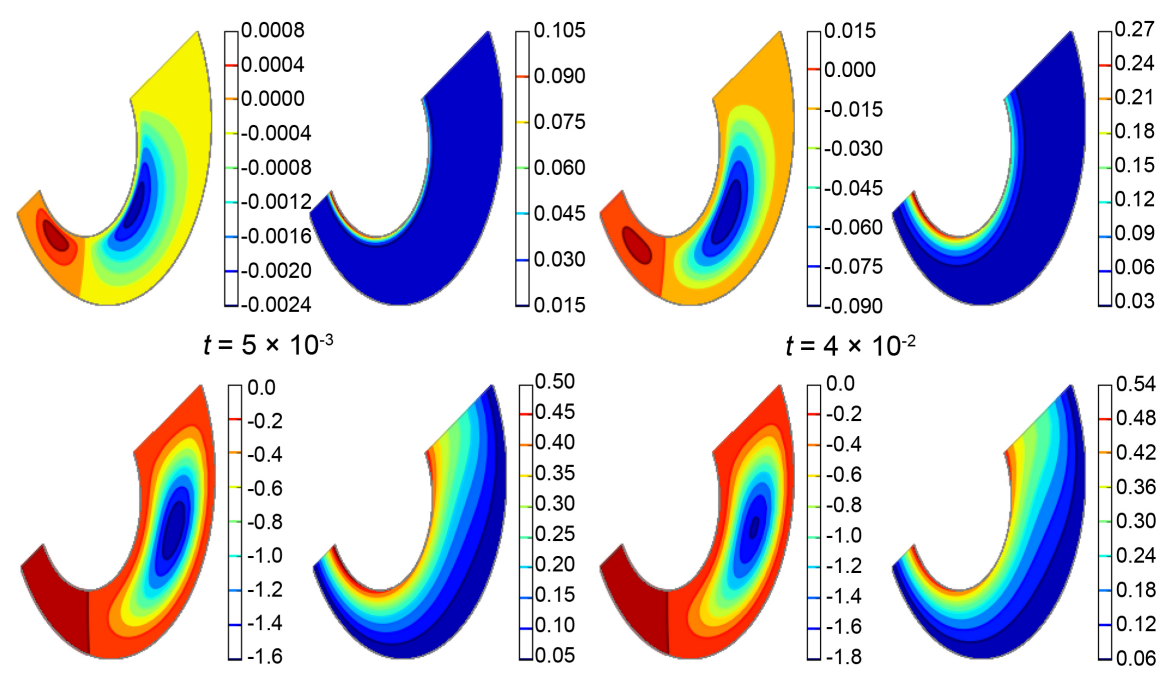

$$
t=4 \times 10^{-2}
$$

$$
t=6 \times 10^{-1}
$$

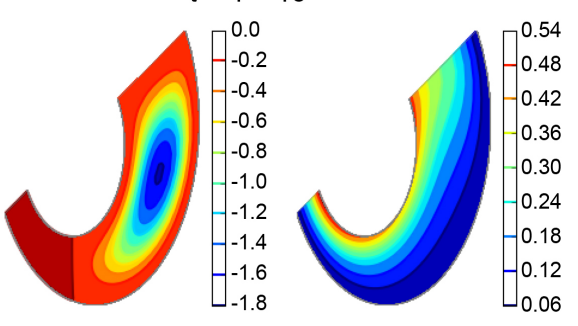

$t=1$

Figure 2. The evolution of streamlines and isotherms for $R a=10^{3}, e=0.5$ and $\varphi=\pi / 3$.

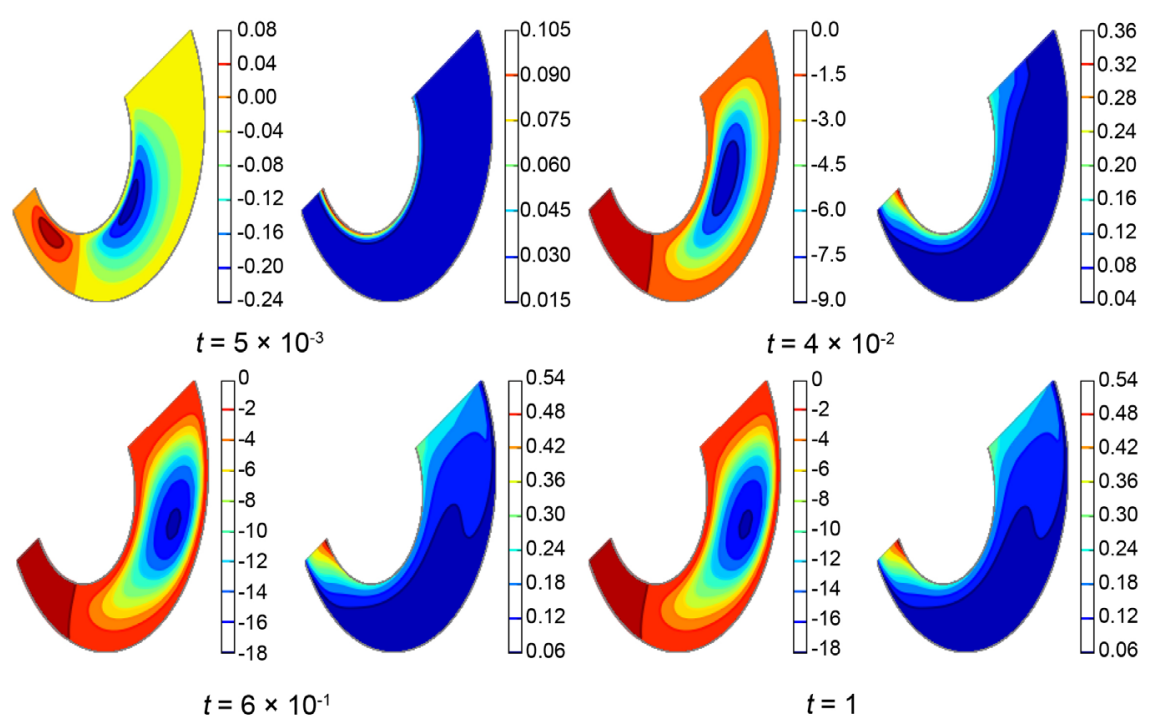

Figure 3. The evolution of streamlines and isotherms for $R a=10^{5}, e=0.5$ and $\varphi=\pi / 3$. 


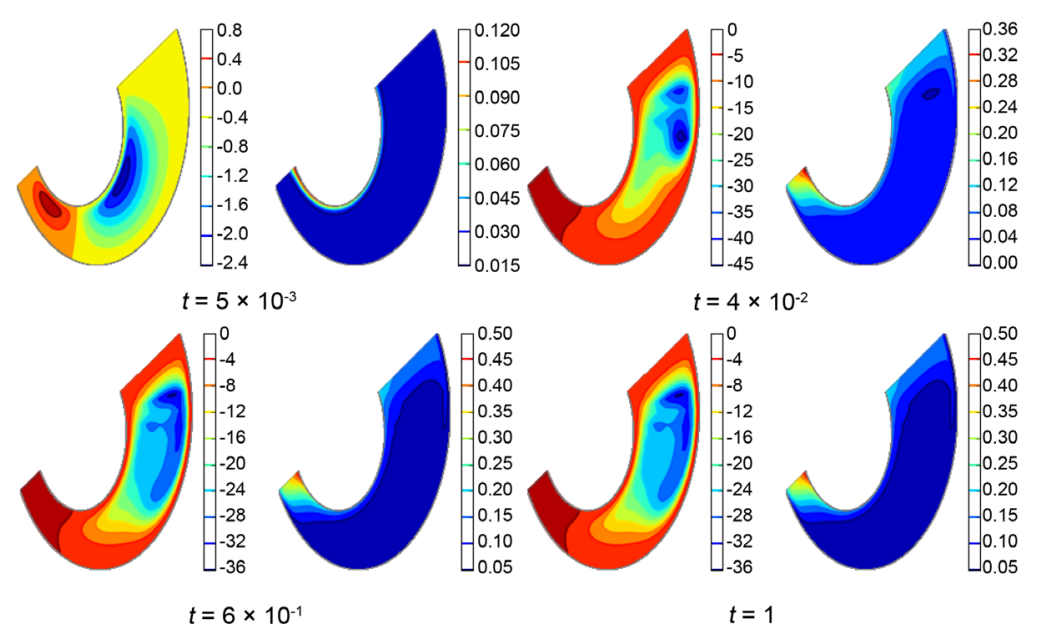

Figure 4. The evolution of streamlines and isotherms for $R a=10^{6}, e=0.5$ and $\varphi=\pi / 3$.

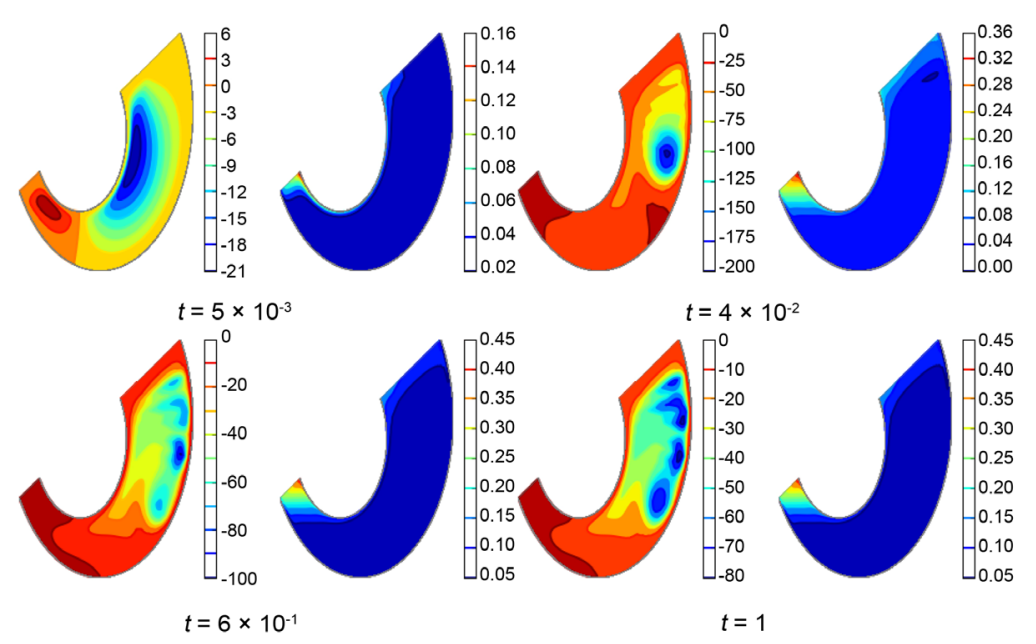

Figure 5. Evolution of streamlines and isotherms for $R a=10^{7}, e=0.5$ and $\varphi=\pi / 3$.

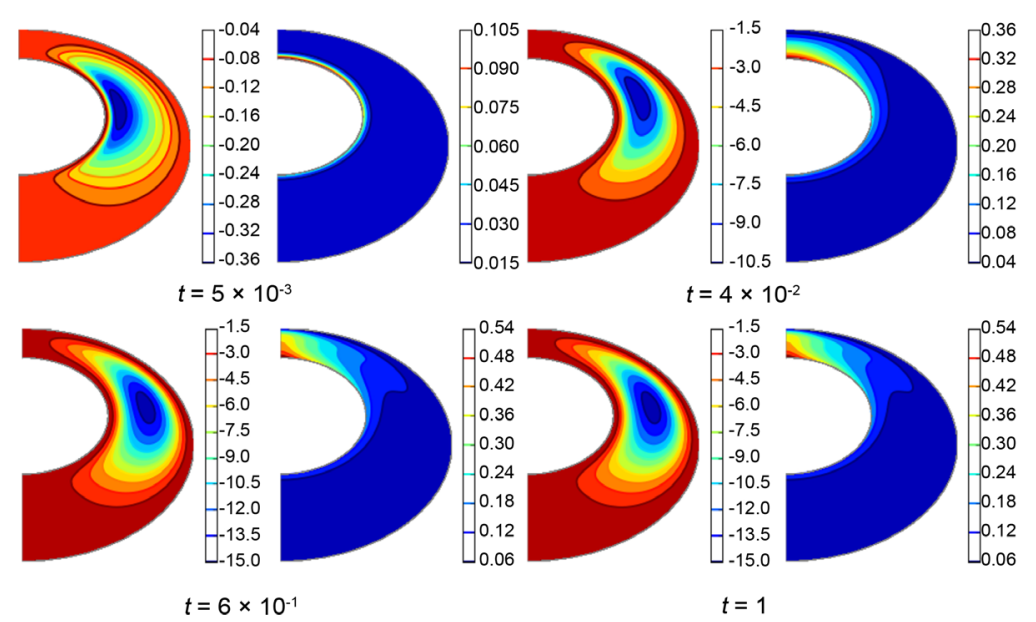

Figure 6. Evolution of streamlines and isotherms for $R a=10^{5}, e=-0.5$ and $\varphi=0$. 


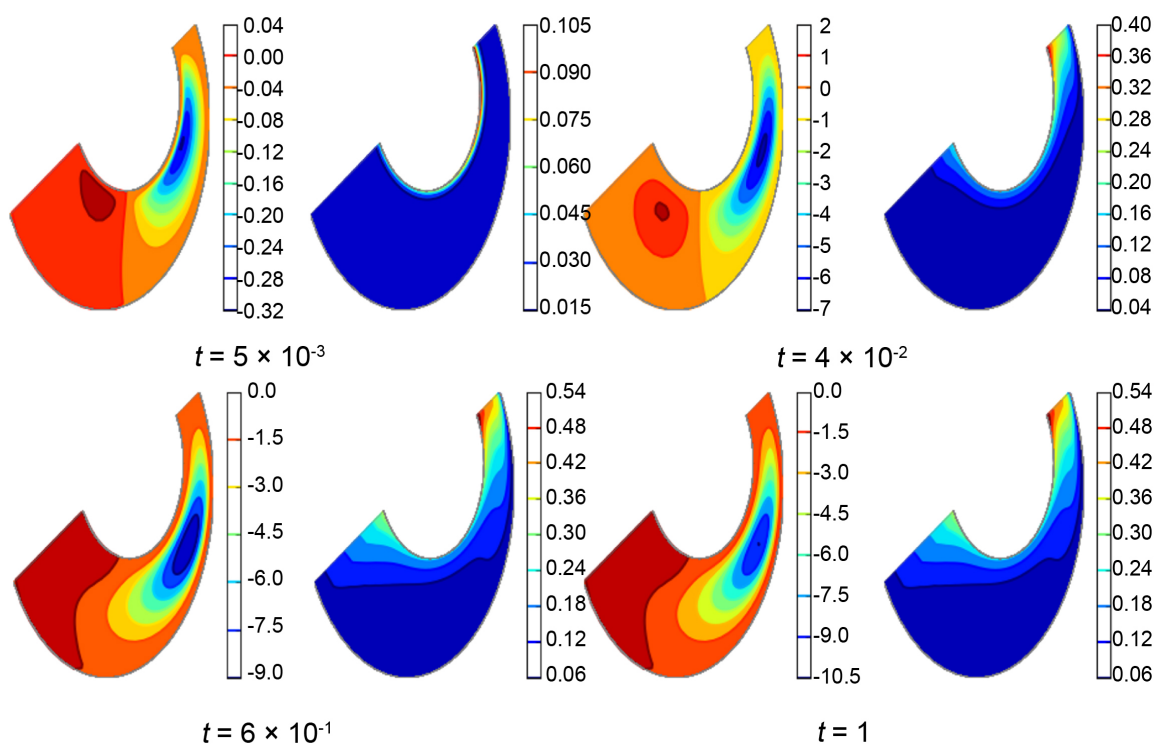

Figure 7. Evolution of streamlines and isotherms for $R a=10^{5}, \quad e=-0.5$ and $\varphi=\pi / 3$.
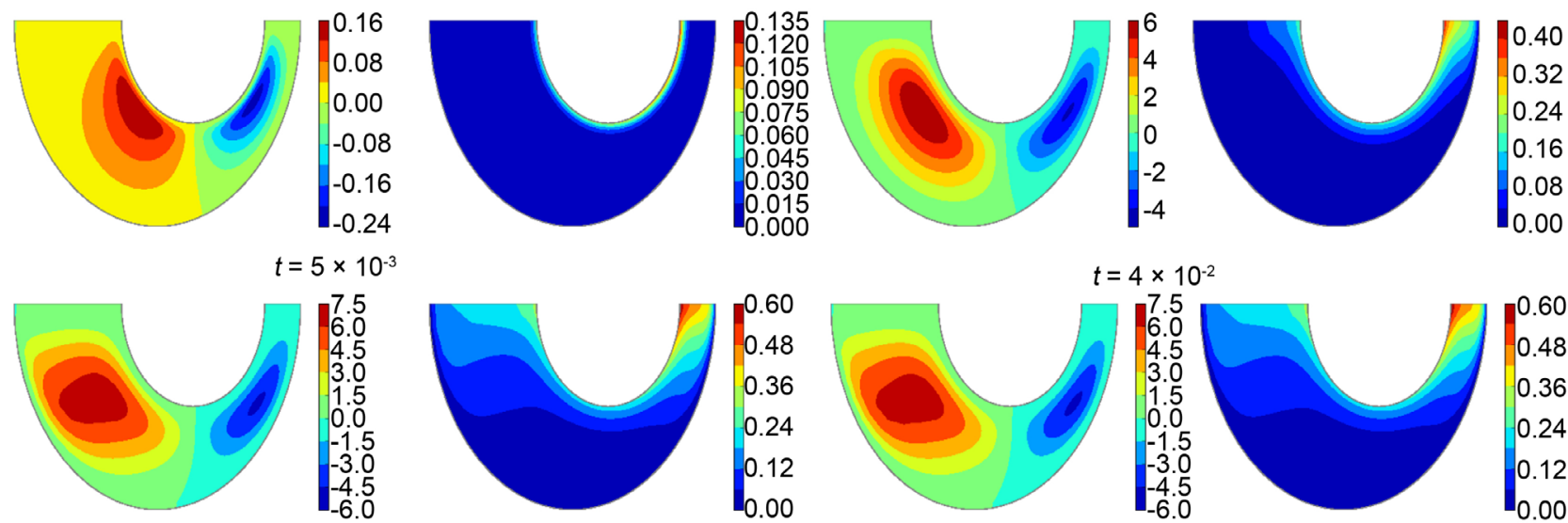

$t=6 \times 10^{-1}$
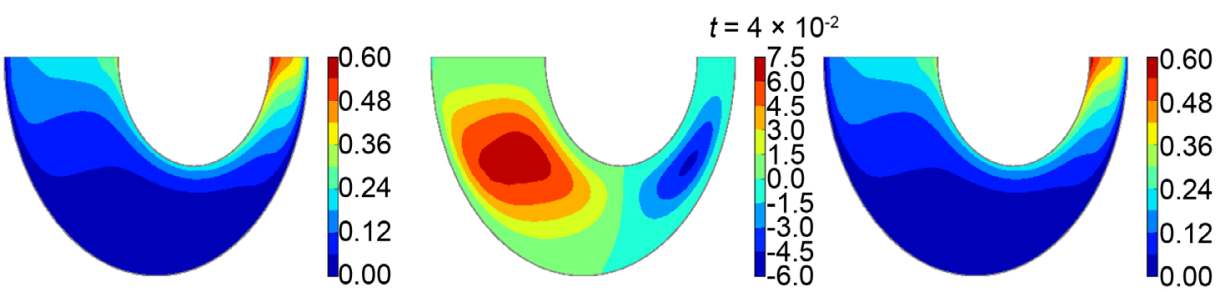

$t=1$

Figure 8. Evolution of streamlines and isotherms for $R a=10^{5}, \quad e=-0.5$ and $\varphi=\pi / 2$.

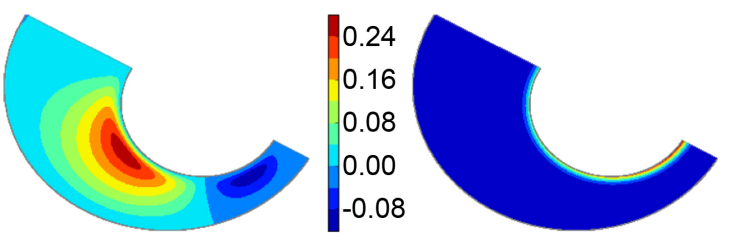

$t=5 \times 10^{-3}$

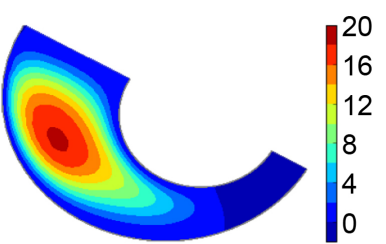

$t=6 \times 10^{-1}$
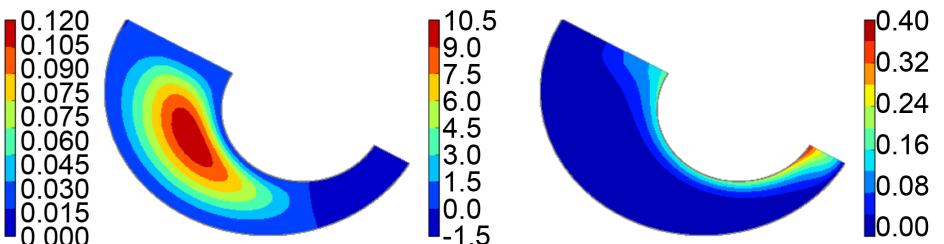

$t=4 \times 10^{-2}$
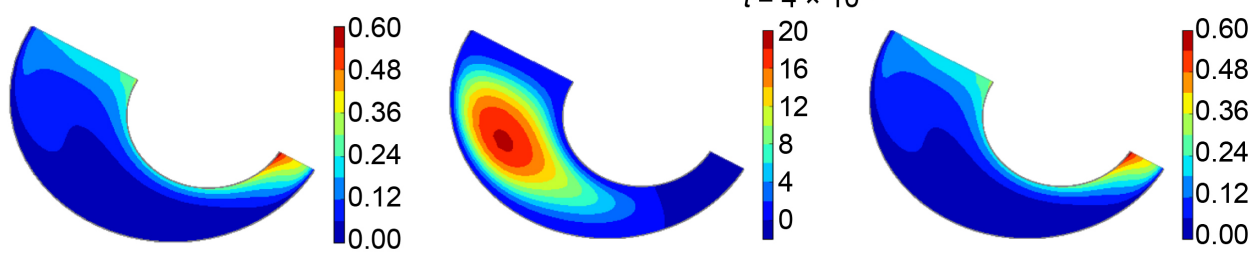

$t=1$

Figure 9. Evolution of streamlines and isotherms for $R a=10^{5}, \quad e=-0.5$ and $\varphi=2 \pi / 3$. 

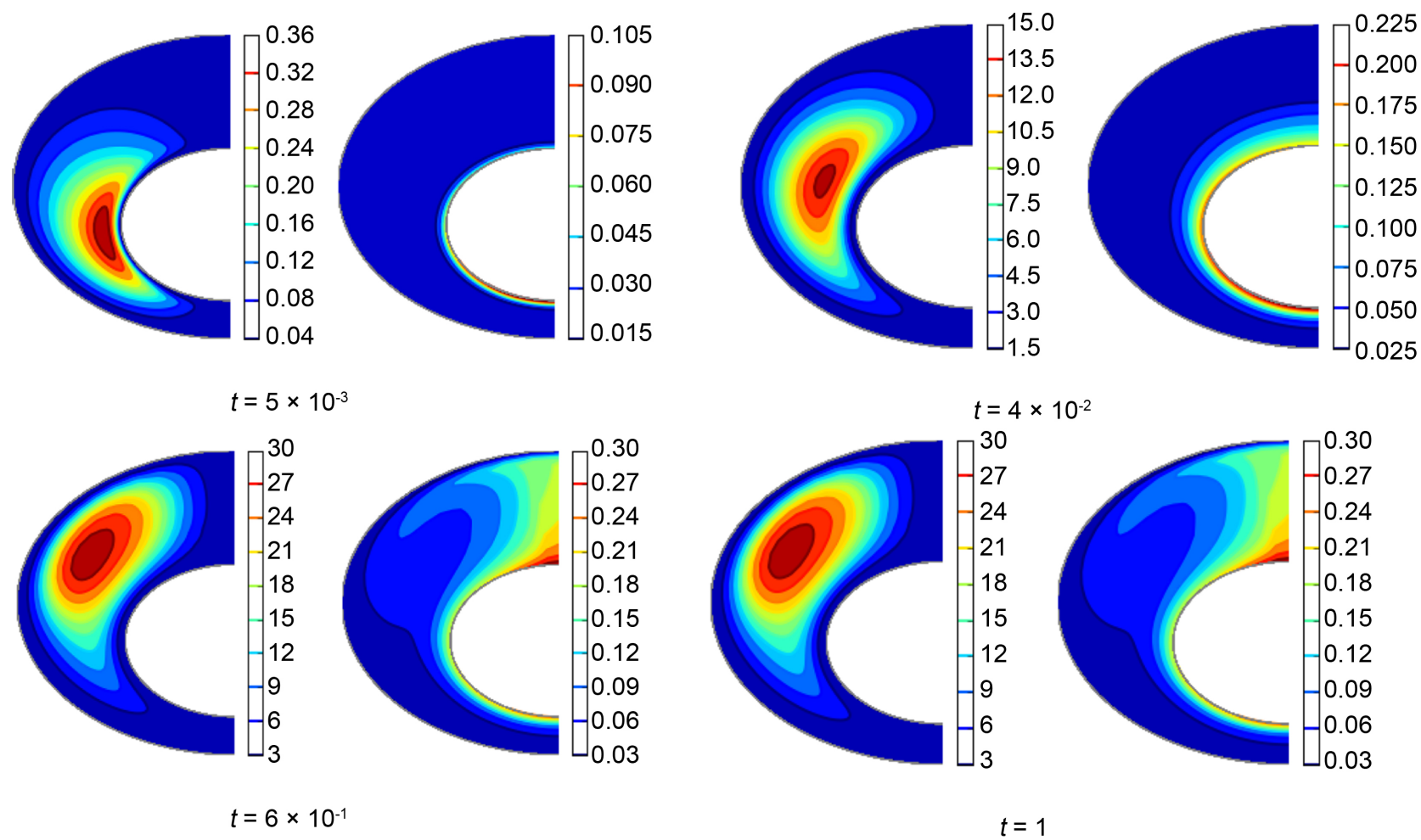

Figure 10. Evolution of streamlines and isotherms for $R a=10^{5}, e=-0.5$ and $\varphi=\pi$.

they occupy almost the entire hemispherical cavity. For the value of angle $\varphi=\pi / 3$ (Figure 7), at the first moments, the flow is constitued by two cells that turns in opposite directions. The secondary cell disappears in favor of the main cell. The isotherms are little deformed and remain localized to the internal wall. At $\varphi=2 \pi / 3$ (Figure 10), the bicellular flow rather vanished over time compared to the value $\pi / 3$. When the value of angle reaches the value $\pi / 2$ (Figure 8 ), the bicellular flow is stable throughout time. The isotherms are very distorded and reached a maximum value compared to other angle values.

Figure 11 present the evolution of Nusselt number, the stream function and the dimensionless of temperature of the heated wall. For the variation of the Nusselt number against dimensionless time for different values of angles, we notice that the value of the Nusselt number is almost the same for the angles $\pi / 3$, $\pi / 2$ and $2 \pi / 3$. On the other hand, we note a difference for the value of $\pi$ and 0 , the value of Nusselt number for $\pi$ is more important and its agrees with isotherms wich were more distorted than those of $\varphi=0$. The same remarks are observed for the variation of the average temperature of heated wall, namely that the values of the temperature for the angles $\pi / 3, \pi / 2$ and $2 \pi / 3$ are equal and are the highest. The value of $\varphi=\pi$ is the lower. The evolution of minimum of stream, it decrease with and the angle of inclinaison.

\subsection{Influence of Eccentricity}

Figure 12 and Figure 13 illustrate streamlines and isotherms respectively for a 


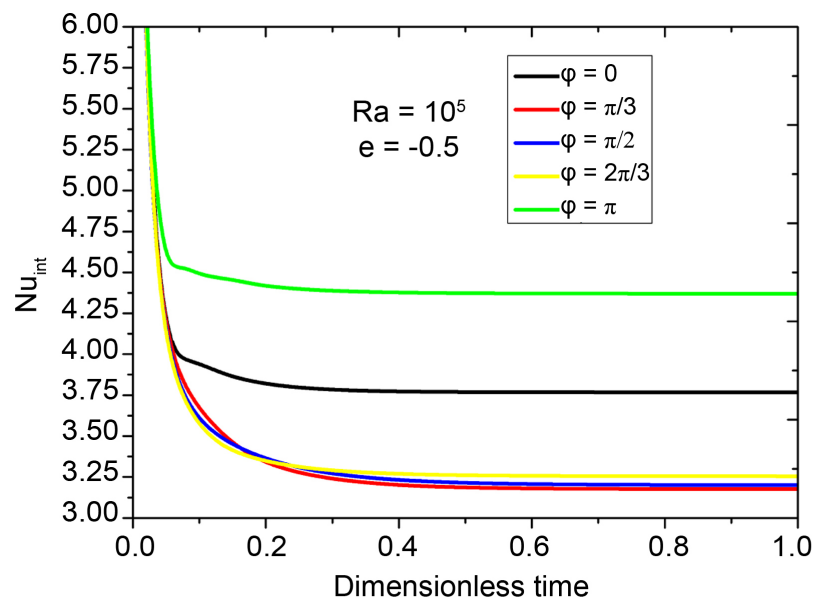

Influence of $\varphi$ on $N u_{\text {int }}$

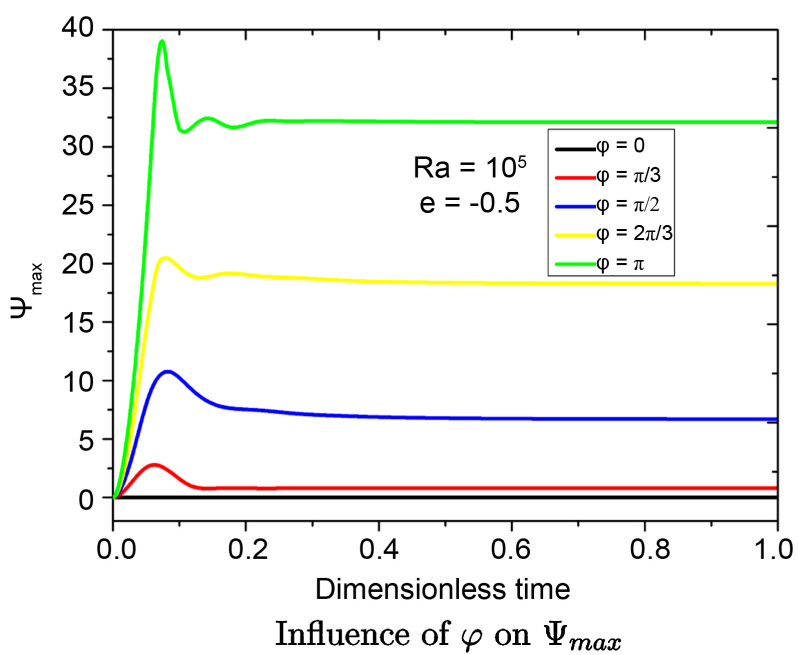

Figure 11. Influence of parameters on $\varphi$.
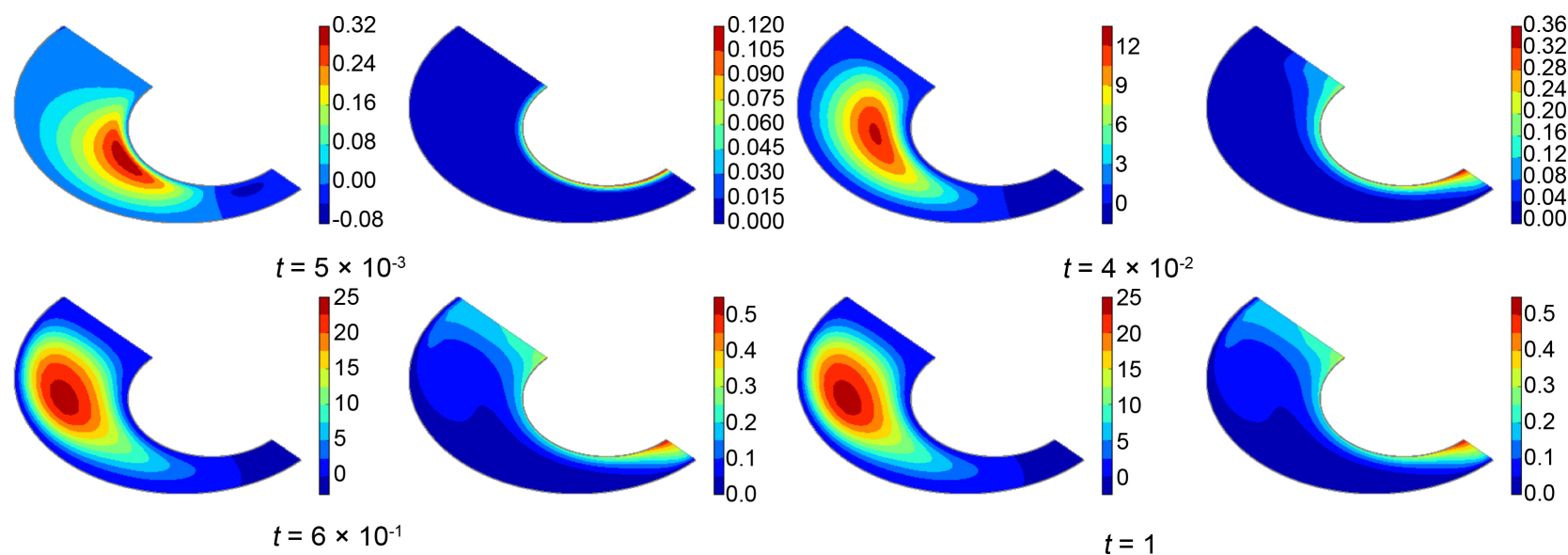

Figure 12. Evolution of streamlines and isotherms for $R a=10^{5}, e=-0.5$ and $\varphi=3 \pi / 4$.

negative eccentricity -0.5 and for a positive eccentricity +0.5 . The streamlines show the monocellular structure appears rather with the negative eccentricity. 


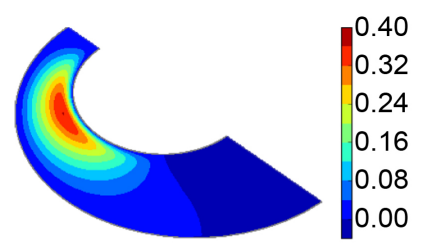

$t=5 \times 10^{-3}$

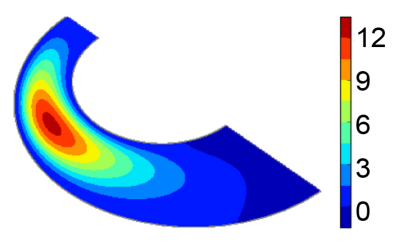

$t=6 \times 10^{-1}$

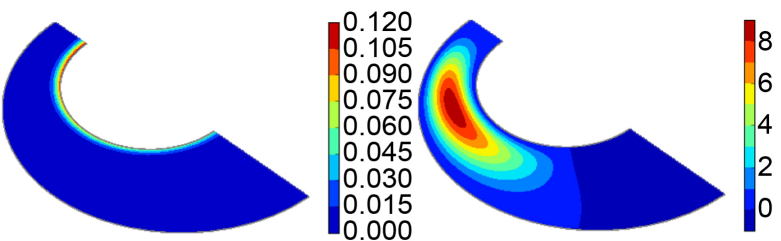

$t=4 \times 10^{-2}$

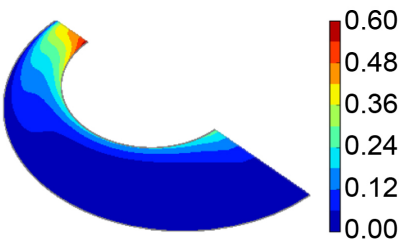

0.60
0.48
0.36
0.24
0.12
0.00

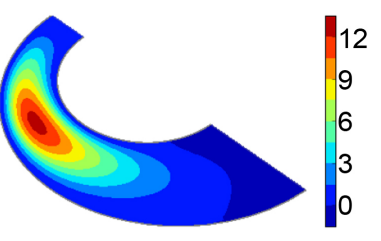

$t=1$
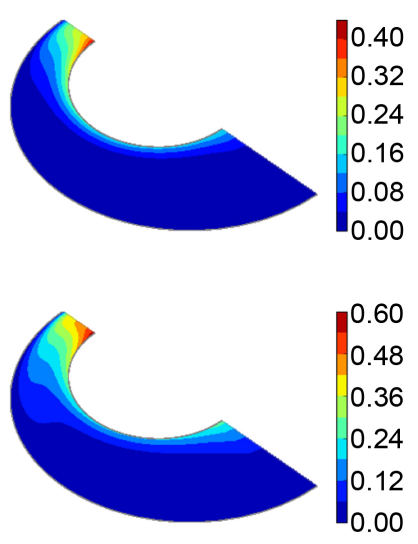

Figure 13. Evolution of streamlines and isotherms for $R a=10^{5}, e=0.5$ and $\varphi=3 \pi / 4$.

Likewise, the isotherms are much more deformed and more fill the cavity for this structure. When the eccentricity increases, we note a decrease in heat exchanges.

\section{Conclusions}

The numerical simulation of transient natural convection between two inclined hemispheres whose the inner hemisphere is subjected to a heat flux of constant density while the outer one is maintened isothermal with time is conducted. The bispherical coordinate and finite difference methods are used.

It emerges from the results presented that when the Rayleigh number is equal to $10^{3}$, heat transfers are dominated by conduction whatever the inclination and eccentricity. The convective transfers intensify as the Rayleigh number increases and there is a strongly destabilized regime for a Rayleigh number equal to $10^{7}$. The simulations have also shown that the topology of the flow is strongly dependent on the inclinaison because the flow can change from a monocellular regime to a bicellular regime by varying the inclinaison. We remark also when the eccentricity increase, we note a decrease in heat exchanges. It would also be interesting to replace the Newtonian fluid with a complex fluid, for example, a fluid whose viscosity depends on the temperature.

\section{Conflicts of Interest}

The authors declare no conflicts of interest regarding the publication of this paper.

\section{References}

[1] Kane, M.K., Mbow, C., Sow, M.L. and Sarr, J. (2017) A Study on Natural Convection of Air in a Square Cavity with Partially Thermally Active Side Walls. Open Journal of Fluid Dynamics, 7, 623-641. https://doi.org/10.4236/ojfd.2017.74041

[2] Bazak, T., Roy, S., Balakrishnan, A.R. and Sarr, J. (2006) Effects of Thermal Boundary Conditions on Natural Convection Flows within a Square Cavity. International 
Journal of Heat and Mass Transfer, 49, 4525-4535.

https://doi.org/10.1016/j.ijheatmasstransfer.2006.05.015

[3] Sarr, J., Mbow, C., Chehouani, H., Zeghmati, B., Benet, S. and Daguenet, M. (1995) Study of Natural Convection in an Enclosure Bounded by Two Concentric Cylinders and Two Diametric Planes. Journal of Heat Transfer, 117, 130-137. https://doi.org/10.1115/1.2822292

[4] Waheed, M.A. (2008) An Approach to the Simulation of Natural-Convective Heat Transfer between Two Concentric Horizontal Cylindrical Annuli. An International Journal of Computation and Methodology, 53, 323-340. https://doi.org/10.1080/10407780701632510

[5] Bishop, M.A. (1966) Heat Transfer by Natural Convection between Concentric Spheres. International Journal of Heat and Mass Transfer, 9, 653-662.

https://doi.org/10.1016/0017-9310(66)90041-X

[6] Sow, M.L., Sarr, J., Mbow, C., Mbow, B., Claudet, B. and Kane, M.M. (2009) Geometrical and Rayleigh Number Effects in the Transient Laminar Free Convection between Two Vertically Eccentric Spheres. International Journal of Numerical Methods for Heat and Fluid Flow, 19, 689-704. https://doi.org/10.1108/09615530910963599

[7] Cabelli, M.A. (1977) Natural Convection in Inclined Hemispherical Cavities. Applied Scientific Research, 33, 45-73. https://doi.org/10.1007/BF00383192

[8] Shiina, Y., Fujimura, A., Akino, N. and Kunugi T. (1988) Natural Convection Heat Transfer in Hemisphere. Journal of Nuclear Science and Technology, 25, 254-262. https://doi.org/10.1080/18811248.1988.9733585

[9] Lewandowski, W.M., Kuski, P., Khubeiz, J.M, Bieszk, H., Wilczewski, T. and Szymanski, S. (1996) Theoretical and Experimental Study of Natural Convection Heat Transfer from Isothermal Hemisphere. International Journal of Heat and Mass Transfer, 40, 101-109. https://doi.org/10.1016/S0017-9310(96)00075-0

[10] Saber , H.H. and Laouadi, A. (2011) Convective Heat Transfer in Hemispherical Cavities with Planar Inner Surfaces. ASHRAE Transactions, 117, 1-32.

[11] Baïri, A. and García de María, J.M. (2013) Numerical and Experimental Study of Steady State Free Convection Generated by Constant Heat Flux in Tilted Hemispherical Cavities. International Journal of Heat and Mass Transfer, 66, 355-365. https://doi.org/10.1016/j.ijheatmasstransfer.2013.07.038

[12] Alilat, N. (2017) Natural Convective Heat Transfer in the Air-Filled Interstice between Inclined Concentric Hemispheres:Application to Thermoregulation in Electronics. International Journal of Numerical Methods for Heat and Fluid Flow, 27, 2375-2384. https://doi.org/10.1108/HFF-10-2016-0392

[13] Koita, M.N., Sow, M.L., Thiam, O.N., Traoré, V.B., Mbow, C. and Sarr, J. (2021) Unsteady Natural Convection between Two Eccentric Hemispheres. Open Journal of Applied Sciences, 11, 177-189. https://doi.org/10.4236/ojapps.2021.112012

[14] Moon, P. and Spencer, D. (1971) Field Theory Handbook: Including Coordinate Systems, Differential Equations. Springer, New York.

[15] Peaceman, D.W. and Rachford Jr., H.H. (1955) The Numerical Solution of Parabolic and Elliptic Differential Equations. Journal of the Society for Industrial and Applied Mathematics, 3, 28-41. https://doi.org/10.1137/0103003

[16] Bejan, A. (1978) Convection Heat Transfer, A. Wiley-Interscience .

[17] Tazi-Charki, M.N., Daoudi, S., Le Palec, G. and Daguenet, M. (1997) Étude numérique du modèle de Boussinesq de convection naturelle laminaire axisymétrique per- 
manente dans un espace annulaire compris entre deux spheres. Revue Générale de Thermique, 36, 239-251. https://doi.org/10.1016/S0035-3159(97)80685-X

\section{Nomenclature}

\section{Grecs symbols}

$\alpha$ Thermal diffusivity, $\mathrm{m}^{2} \cdot \mathrm{s}^{-1}$

$\beta$ Thermal expansion coefficient, $\mathrm{K}^{-1}$

$\eta, \theta, \zeta$ Bispheric coordinate, $\mathrm{m}$

$\lambda$ Thermal conductivity, $\mathrm{W} \cdot \mathrm{m}^{-1} \cdot \mathrm{K}^{-1}$

$\mu$ Kinematic viscosity, $\mathrm{kg} \cdot \mathrm{m}^{-1} \cdot \mathrm{s}^{-1}$

$\Omega$ Dimensionless vorticity

$\Psi$ Dimensionless volume stream function

$\rho$ Density, $\mathrm{kg} \cdot \mathrm{m}^{-3}$

$\varphi$ Angle of inclinaison, rad

\section{Latin letters}

a Characteristic length $a=R_{e}-R_{i}, \mathrm{~m}$

$e$ Dimensionless eccentricity

$g$ Gravitational acceleration, $\mathrm{m} \cdot \mathrm{s}^{-2}$

$G_{1}, G_{2}$ Coefficients

$H$, $K$ Dimensionless parameters

$\mathrm{Nu}$ Nusselt number

Pr Prandlt number

$R a$ Rayleigh number

$T$ Dimensionless temperature $T=\frac{\lambda}{q a}\left(T^{\prime}-T_{0}\right)$

$t$ Dimensionless time

$U, V$ Dimensionless velocity components

$x, y, z$ Cartesian coordinates, $\mathrm{m}$ 